\title{
A Goal-based Framework Integrating Disparate Media Choice Theories
}

\author{
Yoram M. Kalman \\ Department of Management and \\ Economics \\ The Open University of Israel \\ yoramka@openu.ac.il
}

\author{
Keri K. Stephens \\ Moody College of \\ Communication \\ The University of Texas at \\ Austin \\ Keristephens@austin.utexas.edu
}

\author{
Dron M. Mandhana \\ Department of Communication \\ Villanova University \\ dron.mandhana@villanova.edu
}

\begin{abstract}
Media choice and selection theories are numerous and highly fragmented. While much of this theorizing has helped IS researchers better understand what influences people's media choices and selections, the proliferation of theories also leads to redundancies, and decreased clarity and impact. Here, we develop and apply an approach to "better know what we know" about a set of related theories. We present a unifying framework of media choice that (1) builds on prior work, (2) streamlines disparate lines of research, and (3) links media choices to goals. In addition to advancing media choice theorizing, the framework is a useful template for relating future research contributions to previous theories, an effective teaching aid, and a tool for practitioners applying media choice theories.
\end{abstract}

\section{Introduction}

While many different theories have refined concepts of media choice-often in additive or comparative ways-researchers in Information Systems (IS) and in Communication still struggle with knowing how to test these various theories and how to use them to advance the fields. Human communication in general, and message production in particular, are goal directed activities $[1,8]$. In this paper we use the goal construct from the message production research stream to revisit the media choice research stream and propose a unified, goal-based framework of media choice. The unified framework presented here integrates nine different theories, relates them to goals, and suggests ways we can better "know what we know" and advance IS theory.

Most research on message production and goals focuses on the content of the messages, pays little attention to the medium, and is concerned with interpersonal communication [e.g. 14, 18]. By contrast, most research on media choice focuses on the medium people choose and use for conveying their message, and is chiefly concerned with digital media in organizational contexts, and to a lesser extent focuses on interpersonal contexts [35]. Furthermore, media choice research is highly fragmented, is pursued by both IS researchers and Communication researcherstwo research communities that often do not overlap. Consequently, diverse theories such as media richness theory [5] and social information processing [42] are indiscriminately invoked in various contexts regardless of boundary conditions, often outside of the theory's original context [43].

Here, we demonstrate how using a goals perspective can (1) organize and integrate the fragmented media choice research, (2) resolve inconsistencies in that literature, (3) promote new productive research agendas in communication and technology research, and (4) more effectively support the needs of practitioners. In the following section we first present the media choice research thread and its achievements and challenges. Next, we present the goal construct in general, and then specifically in the context of message production research. Following this background section, we demonstrate how the goal construct provides a new perspective on media choice research, and how reframing media choice theories using a goal-based framework can serve to unify these fragmented fields. We conclude with a discussion of the theoretical and applied implications of the proposed framework.

\section{The Backdrop for Creating a Goal- Based Framework of Media Choice}

Meaning is communicated through the exchange of messages. Often the message component-which comprises the meaning - is considered separately from the component which transports the message-the 
"medium." Yet actually, the medium and the message are inseparable constituents of the communication process [32]. Particularly, both the message content and the medium can convey symbolic meaning in many communication situations [32].

Discussions of media choice and media selection have been an integral part of the discourse of Information Systems and Communication research for decades [e.g. 6, 27, 28]. These discussions have consistently underscored the importance of the medium in the communication process. The proliferation of information systems and digital media in the workplace from the 90s and on, exponentially expanded communication options by allowing users to both instantaneously and asynchronously communicate using any combination of text, voice, and combinations of still and moving pictures. This proliferation of options was accompanied by extensive theorizing on media choice and media use.

Literature reviews of media choice research usually begin by identifying two archetypical categories of media choice theories [3, 34, 48]: Theories in the first category focus on the characteristics or traits of different media, and theorize media choice as a rational process of aligning media traits with message content and other situational variables. Media richness theory $[4,6]$ is the prominent representative in this group. Theories in the second category emphasize the importance of social and environmental factors, and theorize media choice as a process that is mainly influenced by social forces. The social influence model of technology (SIT) $[12,13]$ is a prominent representative of this second category.

Theories which discuss media choice and do not fall into one of these two archetypical categories are more difficult to classify. Social information processing (SIP) theory showed that when people want to engage in relational communication, they can use computer-mediated channels to achieve the same quality as face-to-face interactions, given enough time to develop the relationship [42]. Other researchers raised sociomaterial arguments to provide more comprehensive explanations, and argue that scholars must consider both social and material considerations of technologies if we are to understand how humans use media [22, 38]. Straub and Karahanna's [37] work represents one of the first attempts to consider that media choice is not always an individual-level choice. These scholars claim that knowledge workers want to get conversations completed, or close tasks, and this desire motivates their choice of communication media [37]. Building on the idea that closing a communication task can result in needing to send multiple messages, Stephens, Sørnes, Rice, Browning, and Sætre [36] examined specific sequences of information and communication technologies (ICTs) and linked sequences of ICTs to goals. They used information theory [31] and cost minimization theory [23] to explain why people use multiple media to communicate a message. They also used uses and gratifications [17] to provide a list of reasons people use media.

The diversity of these theories resulted in a variety of theoretical viewpoints and approaches being promoted. The research revealed many important variables that influence and are influenced by the way people use media to communicate. Nevertheless, this theoretical expansion was not accompanied by convergence. Having no single agreed upon theoretical base in media choice is to be expected in the social sciences where pluralism is a fundamental principle. Nevertheless, excessive divergence makes it difficult to build a body of research that can be tested empirically and applied by practitioners, and opportunities for unifying theories (e.g. UTAUT - the Unified Theory of Acceptance and Usage of Technology [41]) should be explored. Furthermore, researchers often use these theories imprecisely. For example, much of the research on media richness grabs only part of original information richness theory and makes the assumption that face-to-face communication is inherently better than less rich media. The complete theory is more about efficiency and matching the task and medium.

In contrast with the theoretical approaches described so far, which focused on augmenting and extending the foundational media choice theories, or on developing alternative theoretical approaches, another group of studies attempted to reframe media choice and use concepts in ways that overcame limitations that were raised when the foundational theories were tested empirically. These integrative studies suggest that some of the older theoretical approaches such as media richness theory and the social influence model of technology are actually not competing, but rather they complement one another and apply in different situations. For example, media use differences often exist between groups of employees [3], categories of media [48], or purposes, contexts or communicative goals [16, 26, 30]. This current conceptual paper continues and extends the integrative approach used in these types of empirical and theoretical research. Instead of choosing one media choice theory over another, our approach proposes an inclusive framework that can accommodate many theories. Furthermore, the framework we develop demonstrates that most of the apparently divergent theories can be unified under a common framework, a goal-based framework. 


\subsection{The Goal Construct}

The term goal is commonly used in everyday life, as well as being a concept employed in many theoretical perspectives in the behavioral and social sciences $[8,25]$. It is generally accepted that human communication in general, and message production in particular, are goal directed activities, and researchers have devoted extensive resources to understand goals and their role in communication [25]. Research findings have demonstrated that goals provide a simple perspective that helps people observe and study human communication [8]. On the other hand, as the study of goals has evolved, it has become evident that the goal construct itself, although perceived to be simple, is complex and multi-faceted. In fact, it is this combination of simplicity and complexity that is likely at the heart of the successful applications of the goal construct in social theorizing. This is demonstrated in heavily cited work stating that "...language is a tool or an instrument for attaining goals" [1:49], and that "goals provide a parsimonious means for summarizing social reality" [8:97]. Viewing human communication through the perspective of goals provides researchers a tool that is simple, but does not ignore the complex context of social life in which communication is occurring.

What is a goal? Dillard's [8] discussion of the goal construct suggests that although there is growing consensus on the utility of the construct, its definition varies significantly between studies. A recent discussion of the goal construct suggests that goals be defined as "mental representations of a desired endstate" [24:81]. This is a useful definition, but there are caveats. For example, Palomares [24] emphasizes the distinction between goals and behaviors, and between goals and constraints. Dillard emphasizes several key components of goals that are helpful for our framework development. People are not always consciously aware of goals, their commitment to goals can vary, and the level of specificity of goals can impact many goalrelated considerations. Goals also have hierarchical relationships between them and they can have subcomponents. Dillard [8] identified differences between specific types of goals, such as approach and avoidance goals, and between process and outcome goals. Furthermore, in Dillard and Solomon's [10] discussion of context in message production research, they state that "goal structures generally provide succinct summaries of the complex, multi-variate configurations that define distinct message-production contexts" (p.173), thus suggesting that the same goal in a different context is actually a different goal.

The nomenclature we use in this paper combines Dillard's Goals-Planning-Action (GPA) model [9] and the uses and gratification (UGT) model [17], adapting Vance, Wilson, and Lu's [40] approach in their study of communication goals and online persuasion. The communicator has a primary, instrumental goal that is based on the benefits s/he desires to achieve and the costs $\mathrm{s} / \mathrm{he}$ desires to avoid. This primary instrumental goal guides the communicator, while secondary goals influence that behavior. The secondary goals, or influencers, play an important role in our model, so we focus on four main categories of secondary goals:

- Identity goals influence the communicator's behavior so they align with an individual's self-concept including ethical, moral, and personal standards.

- Interaction goals influence the extent to which the communicator's behavior aligns with socially appropriate behavior (e.g., impression management and face).

- Arousal management goals influence the communicator's behaviors related to a person's preferred affective states, such as levels of anxiety or anger.

- Relational resource goals influence the communicator's behavior, and the extent to which the behavior aligns with a person's desired social and personal relationships.

By examining media choice and media selection theories from a goal-based perspective, this framework builds on the notion that human communication is an instrument for attaining goals. Furthermore, when messages are produced, the desired end-state will have a strong impact on the message. As the number of media available to people increases, media choice, or the form of the message, becomes an increasingly important aspect of message production. For example, Jung and Lyytinen [16] demonstrate that media choice is a dynamic multi-dimensional process where users identify the media affordances that will help them achieve their communication goal in their specific context. Similarly, a genre analysis method for team communication analysis proposed by Riemer and Filius [26] identifies genres by coding and grouping the observed communication acts according to the purpose of the acts for the team, or, in other words, the team's goals. Thus, linking media choice to goals is a natural extension of the well-established research on message production, and is in line with recent media choice research literature.

Since most message production research is undertaken in the interpersonal communication context, and since context is, as explained above, an integral aspect of the social reality that goals summarize, context should be noted alongside goals $[16,26]$. For example, if a communicator's main goal is to get another person to help her, that goal will influence her media choice (and her message 
production in general) differently depending on whether the person is a loved family member, or a work colleague. The goal of getting assistance in a family context is different from the goal of making a positive impression in a workplace context.

\section{A Goal-based Framework for Media Choice Research}

Having reviewed the media choice research threads and the goal construct, we now present our core thesis, which is that a goal-based framework can unify many of the media choice theories. We suggest that despite the significant differences between the theories, they can all be viewed as theories in which the choice of medium (or media) is the dependent variable, while the key independent variable is the main goal of the communicator. Accordingly, our analysis of each media choice theory identifies the additional independent variables that researchers included in their models of media choice (some of which can be classified as secondary goals), as well as the context of the communication. Our work continues the integrative approach to media choice theories $[3,16,26,30,39$, 48 ], and it invites researchers to build on the examples we provide.

To illustrate the value of our approach, we use the goal-based framework to analyze nine theories that discuss media choice. We illustrate how our framework integrates theories, by identifying (1) the primary, instrumental goal, (2) other independent variables that influence media choice, and if relevant (3) we classify independent variables that fall into one or more categories of the four secondary goals. Finally, our analyses identifies the context in which the theory was developed: organizational and/or interpersonal. Table 1 summarizes the analysis. Due to space limitations, the table uses the acronyms or abbreviations listed in parentheses in sections 4.1-4.9. For a full unabridged table see https://tinyurl.com/HICSS2019 .

\subsection{Media Richness Theory}

Media richness theory views organizations as information processing units and organizations' successes are dependent on their employees' abilities to accomplish tasks by communicating information effectively $[5,6]$. This theory focuses on the instrumental goals of accomplishing tasks (AT), coordinating diverse activities (CA), and interpreting the environment (IA) [6]. The theory states that managers' effectiveness is determined by how appropriately they match a medium to a task.
Specifically, managers should use lean media, such as written documents, to communicate unambiguous information, and rich media, such as the phone or faceto-face conversation, to communicate ambiguous information. Thus, the other independent variables that influence media choice are the ambiguity of the information that needs to be communicated (Am), and the richness of the medium (Rich). Media richness theory does not consider secondary goals. The theory was developed in the context of organizational communication.

\subsection{Media Synchronicity Theory}

Media synchronicity theory suggests that the primary goal of workers communicating in an organizational context is to achieve high levels of communication performance. It explains that although accomplishing a task might be the ultimate goal of the workers, to achieve the task, people need to employ a mix of different communication processes. These individual processes form the necessary steps to accomplish the tasks [7]. Thus, instead of focusing on variables that influence task accomplishment, this theory posits communication performance $(\mathrm{CP})$ as the primary goal of the communicator. The theory suggests other independent variables that influence media choice are (1) the fit between the amount of convergence and conveyance required for high performance $(\mathrm{C} / \mathrm{C}),(2)$ medium synchronicity (MS), and (3) appropriation factors (AF) that include familiarity, training, past experiences, and social norms. The social norms are classified as identity and interaction (secondary) goals. Media synchronicity theory was developed in the context of organizational communication.

\subsection{Social Influence Model of Technology}

The social influence model of technology (SIT) $[12,13]$ has the primary goal of task accomplishment (TA) in an organizational context. It suggests that media choice is influenced by media and task evaluations, and that these evaluations are influenced by both rational choice processes, as well as by social influences. Social influence is mainly expressed in identity and interaction goals (through statements, attitudes and behaviors of coworkers regarding appropriate media use). The other independent variables that influence media choice are personal media evaluations (ME), media experience and skills (ME/S), task evaluations (TE), and general situational factors (SF) such as individual differences, facilitating factors, and constraints. 


\subsection{Social Information Processing Theory}

Social information processing (SIP) theory focuses on the development of interpersonal impressions and relational communication via CMC [44]. Hence, the instrumental goal of the communicators is impression formation (IF). The theory states that the formation of these impressions using CMC takes longer than impressions formed during face-to-face communication. However, given enough time, and exchanges, the relational communication goal can be achieved equally well by using either CMC or face-toface communication. Thus, the other independent variable present in SIP is time (Ti), or the number of exchanges (\#E). Interestingly, the main, instrumental goal of fostering an impression is also a relational source goal, which is one of the four secondary goals in our analysis. In SIP this goal takes center stage. The theory was developed in the context of interpersonal communication, but has since been studied in numerous contexts including organizational communication, online games, and online education.

\subsection{ICT Succession Theory}

ICT succession theory [33] expands media choice theories beyond the choice of one medium to complete one task. Efficient task completion (TC) is the primary instrumental goal. Its unique contribution is that it points out that task completion often requires a succession (two or more) of communication acts, and that these acts might include choosing more than one medium. Thus, one of the key independent variables that influence media choice is the media that previously have been used to achieve the primary goal (PM), and whether or not the goal has been achieved. The theory also posits many other independent variables including task and media traits (T/MT), social and organizational influences (SOI), and individual differences (ID). It does not discuss secondary goals, and focuses on the organizational context.

\subsection{Channel Expansion Theory}

Channel expansion theory was primarily developed to reconcile inconsistent findings in research on media richness theory $[2,11]$. The instrumental goal the theory discusses is communication effectiveness (CE) [2] and these authors argue that any medium can be perceived as rich or lean based on four independent variables: the users' experience with that medium (or channel) (MEx), their experience with the message topic (TEx), experience with their communication partner (PEx), and experience with the specific organizational context (OCEx) in which the communication is occurring. Similar to media richness theory, channel expansion theory does not include secondary goals. The theory was developed in the organizational communication context.

\subsection{Media Naturalness Theory}

Kock [19] proposed media naturalness theory as an alternative to media richness theory that is compatible with social theories such as the social influence model of technology. The theory extends research on the evolution of human behavior. It suggests that human evolution led to the optimization of co-located and synchronous human communication modes which use facial expressions, body language, and sounds, i.e. face-to-face $(\mathrm{FtF})$ communication. It further suggests that other modes of communication which do not fully match the human biological communication apparatus (that has been optimized for FtF communication) will (a) require increased cognitive effort from the communicators, (b) contain communication ambiguity, and (c) decrease physiological arousal. Thus, the primary instrumental goal is the same as that of media richness theory, but three secondary goals are also emphasized: interaction goals, arousal management goals, and relational resource goals. The main additional independent variable is the medium's naturalness $(\mathrm{Na})$, which is defined using five elements: (1) co-location; (2) synchronicity; ability to convey/observe (3) facial expressions (4) body language, and (5) speech.

\subsection{Warranting Theory}

Walther and Parks [46] propose that the warranting value of information is "derived from the receiver's perception about the extent to which the content of that information is immune to manipulation by the person to whom it refers" (p. 552). Warranting theory explains that media choice is influenced by the instrumental goal of impression formation (IF). Individuals' online self-presentations can be predicted to hold higher or lower warranting value based on the type of media used for presentation [46, 47]. For example, information provided by a job seeker on his/her company's website may have higher warranting value than that of the same information provided on the job seeker's own webpage. Walther and Parks [46] state that warranting cues (or warrants) are the independent variables that can provide insights that help individuals in appraising the warranting value (WV) of the information provided online. This theory does not discuss other secondary goals. Warranting theory is 
most often used in an interpersonal communication context, but it has been used in an organizational communication context as well.

\subsection{Theory of Electronic Propinquity}

Korzenny [20] proposed the theory of electronic propinquity to explain the effects of several factors related to media, users, task, setting, and availability of other media, on the electronic propinquity construct. The theory focuses on the instrumental goals of communication effectiveness (CE) and task accomplishment (TA) [45], and does not discuss secondary goals. Korzenny further explains that three factors (independent variables) increase electronic propinquity: (a) the bandwidth of the communication medium (BW), (b) the capacity of the communication channel for mutual directionality (CMD), and (c) the communication skills of the individual communicators (CS). In addition, three factors that decrease electronic propinquity include, (a) the complexity of the information being exchanged (Co), (b) the perceived number of communication rules to which the individuals must conform (CR), and (c) the perceived number of choices among communication channels available to the individuals (CC). Walther and Barzova [45] state the theory originally focused on group communication in organizational contexts, but can be applied to human interactions over a wide range of channels.

Table 1 summarizes the analysis of these nine media choice theories using the goal-based framework. The table demonstrates that the different theories focus on a diverse set of primary goals (e.g., impression formation, task accomplishment, efficiency), that they vary in the attention they give to secondary goals (how many and which ones), and that they include myriad additional independent variables. Furthermore, it details the contexts in which the different theories were formulated.

The results of our analysis provide an organizing framework for theoretical work that spans three decades, that represents diverse disciplinary and methodological orientations, and that studied a variety of communication media, in assorted contexts. The framework can be used as a template for relating future theoretical contributions to previous theories, an aid for teaching about media choice, and as a checklist for independent variables related to media choice. These and other contributions of the study are discussed next.

\begin{tabular}{|c|c|c|c|c|c|c|c|}
\hline & & Sec & ond & ary go & als & & \\
\hline $\begin{array}{l}\text { Theory (see } \\
\text { sections 4.1- } \\
\text { 4.9) }\end{array}$ & \begin{tabular}{|l|} 
Primary \\
instrumental \\
goal
\end{tabular} & Id & In & AM & $\mathbf{R R}$ & $\begin{array}{l}\text { Independent } \\
\text { variables }\end{array}$ & Context \\
\hline $\begin{array}{l}\text { Media } \\
\text { richness }\end{array}$ & AT, CA, IA & $\mathrm{N}$ & $\mathrm{N}$ & $\mathrm{N}$ & $\mathrm{N}$ & Am, Rich & $\mathrm{O}$ \\
\hline $\begin{array}{l}\text { Media } \\
\text { Synchronicity }\end{array}$ & CP & $\mathrm{Y}$ & $\mathrm{Y}$ & $\mathrm{N}$ & $\mathrm{N}$ & $\mathrm{C} / \mathrm{C}, \mathrm{MS}, \mathrm{AF}$ & $\mathrm{O}$ \\
\hline \begin{tabular}{|l} 
Social \\
Influence \\
\end{tabular} & TA & $\mathrm{Y}$ & $\mathrm{Y}$ & $\mathrm{N}$ & $\mathrm{N}$ & \begin{tabular}{l|} 
ME, ME/S, \\
TE, SF
\end{tabular} & $\mathrm{O}$ \\
\hline SIP & IF & $\mathrm{N}$ & $\mathrm{N}$ & $\mathrm{N}$ & $\mathrm{Y}$ & Ti, \#E & In \\
\hline \begin{tabular}{|l} 
ICT \\
Succession \\
\end{tabular} & $\mathrm{TC}$ & $\mathrm{N}$ & $\mathrm{N}$ & $\mathrm{N}$ & $\mathrm{N}$ & $\begin{array}{l}\text { PM, T/MT, } \\
\text { SOI, ID }\end{array}$ & $\mathrm{O}$ \\
\hline \begin{tabular}{|l|} 
Channel \\
Expansion \\
\end{tabular} & CE & $\mathrm{N}$ & $\mathrm{N}$ & $\mathrm{N}$ & $\mathrm{N}$ & \begin{tabular}{|l} 
MEx, TEx, \\
PEx, OCEx
\end{tabular} & $\mathrm{O}$ \\
\hline $\begin{array}{l}\text { Media } \\
\text { Naturalness }\end{array}$ & AT, CA, IA & $\mathrm{N}$ & $\mathrm{Y}$ & $\mathrm{Y}$ & $\mathrm{Y}$ & $\mathrm{Na}$ & $\mathrm{O}$ \\
\hline Warranting & IF & $\mathrm{N}$ & $\mathrm{N}$ & $\mathrm{N}$ & $\mathrm{N}$ & WV & $\mathrm{O} ;$ In \\
\hline $\begin{array}{l}\text { Electronic } \\
\text { Propinquity }\end{array}$ & CE, TA & $\mathrm{N}$ & $\mathrm{N}$ & $\mathrm{N}$ & $\mathrm{N}$ & \begin{tabular}{|l|}
$\mathrm{BW}, \mathrm{CMD}$, \\
$\mathrm{CS}, \mathrm{Co}, \mathrm{CR}$, \\
$\mathrm{CC}$
\end{tabular} & $\mathrm{O} ; \mathrm{In}$ \\
\hline
\end{tabular}

Table 1: Goal-based analysis of media choice theories. Note. Secondary goals abbreviations: Id: Identity, In: Interaction, AM: Arousal Management; RR: Relational Resource. Context: O: Organizational; In: Interpersonal. Other acronyms and abbreviations are noted in the text in sections 4.1-4.9 which analyze each theory. To overcome space and formatting limitations, a version of the table that is not based on acronyms and abbreviations is provided in the link https://tinyurl.com/HICSS2019.

\section{Discussion}

\subsection{Uses of the Goal-based Framework}

This framework provides a structured template that can be used to understand relationships between new research and the existing aggregated knowledge surrounding media choice. For example, new empirical findings about the influence of independent variables on media choice can be compared and contrasted with the current list of independent variables which describes what we already know about media choice. Second, the framework can accommodate novel findings about media choice relating to communication media that have not yet been studied, or that have not existed in the past when the original research was conducted. Third, the framework can be augmented with any new media choice theory. It is interesting to note that we did not come across theories that discuss media choice and which we were not able to analyze using the goal-based framework. Initially, it might seem like our framework applies only to theories which explicitly discuss goals, and not to theories that treat media choice as habitual or "mindless". In fact, while people can use media habitually, and even 
mindlessly, there is evidence that even these types of media behaviors can be related to tasks and goals [21].

The framework's template can also be used as an aid for theoreticians, as it brings together-in a compact and structured manner-disparate theories. For example, SIP theory, which is often cited and successfully used by Communication scholars, is rarely acknowledged by Information Systems (IS) scholars. Our framework presents SIP theory in a context where scholars from related fields can see value in additional study.

Another promising use of the framework is as a teaching aid. It is our experience that teaching undergraduate and graduate students about media choice is often challenging due to the large number of theories and the disjointed nature of the different theories. The goal-based framework can aid students in organizing the different theories in a coherent manner, for example by clarifying similarities and differences between the theories.

The column of independent variables in Table 1 is a useful list of all of the independent variables that we uncovered in the media choice-related theories we analyzed. Despite it being an incomplete list, identifying these independent variables is valuable for anyone interested in a compact summary of variables that existing research has identified as influencing media choice. The list can serve, for example, as a checklist for practitioners who wish to review potential issues surrounding unexpected or ineffective media use in organizations. Similarly, the list can help researchers analyze research scenarios using the goal-based framework instead of having to choose multiple theories.

Finally, this goal-based framework can serve as an example for the IS field, as well as for other disciplines in the social sciences, for an approach to better "knowing what we know" [15]: It integrates and synthesizes a host of theories, demonstrates a way to overcome the lack of standardization for constructs and concepts across fields, and integrates theories in a manner that promotes meta-theorizing [15]. Such a unifying framework can be attempted for other theory groups such as theories of technology acceptance and adoption.

\subsection{Links with Other Theories}

Our framework was inspired by Social Information Processing (SIP) theory [42]. SIP theory, one of the more successful computer-mediated communication theories, effectively explains many of the discrepancies between the various "cues filtered out" theories and actual user behavior. This goal-based framework adopts SIP theory's functional approach to human communication, the approach which proposes that communicators use whatever means they have at their disposal to foster and detect impressions effectively and to achieve their relational resource goals [44].

The framework we propose here also has some commonalities with the uses and gratifications theory (UGT) [17]. This link has been presented in our earlier discussion of the goal construct and it is reflected in the nomenclature we use in this paper. Indeed, UGT is also a goal-oriented theory that explores the media choices made by audience members. UGT mainly is focused on understanding mass media, and it emphasizes the consumption of mass media. Our media choice framework is focused on the production side where users choose a medium or media that will best convey their message, and thus help them achieve their goals. The success of UGT underscores the importance of considering goals as an explanatory variable in human behavior, in general, and in communication in particular. Furthermore, this link between UGT and our framework points to a possible theoretical convergence around the questions of media choice in interpersonal, organizational, and mass communication, on both the production and the consumption side of media use.

\subsection{Future Directions for Research}

The first direction for future research is focused on identifying gaps, duplications and inconsistencies in existing studies. For example, the table shows that most theories ignore secondary goals altogether, while others demonstrate the prominent role of these secondary goals: interaction goals (three theories), identity goals and relational resource goals (two theories each) and arousal management goals (one theory). Accordingly, the fact that only one out of the nine theories takes into account arousal management suggests that scholars should consider whether this secondary goal is important for advancing media choice research. For example, researchers have demonstrated that switching between face-to-face and CMC could be a way to manage emotions [29]. Future findings might demonstrate that either arousal management goals are only marginally relevant for media choice, or, on the contrary, that they have been unjustifiably ignored by most researchers.

The second pathway for research uses the framework to hone in on understudied concepts, variables, and contexts. For example, the framework demonstrates which theories have only been examined in one specific context, thus marking the potential for empirically testing the theories in additional contexts. This framework can also be used to relate findings of new empirical research to previous research. It can 
assist in the formation of new hypotheses, for example, researchers might realize that certain secondary goals or independent variables, already identified as important for message production, should be included in their studies as well. Finally, it is important to note that Table 1 only maps the key foundational papers of each theory, but the same framework can be used to compare and contrast these foundational studies with additional research related to each of the individual theories.

A third pathway forward is using the framework as an organizing tool of existing research. For instance, it could be used to identify the state-of-the-art of research on a specific variable in an identified context. For example, the table demonstrates that if scholars or practitioners are interested in the impact of the experience users have had with a medium in an organizational context, the two theories to focus on are channel expansion theory and the social influence model of technology. The table also identifies the inconsistencies, as well as the lack of theoretical clarity, concerning the exact goals a theory discusses. When viewed through the goals perspective, it becomes clear that too often competing theories are comparing apples to oranges when they compare the variables that influence media choice. Specifically, we see that some studies examine individuals making media choices in the context of one primary goal, while other studies examine media choices of individuals focusing on a different primary goal.

Finally, the goal-based framework and Table 1 lead to a new and noteworthy theoretical question that requires extensive research and consideration well beyond the scope of this paper. The question is whether there exists an ultimate and stable version of Table 1 that future theoreticians and practitioners will be able to consult when they want to know the key variables that influence media choice in a given situation. In other words, will the findings of media choice research eventually converge into an overarching goal-based theory? An affirmative answer to this question suggests that we will ultimately identify the key variables that influence human media choice. Furthermore, an affirmative answer will mean that these variables will remain relatively stable even in an ever-changing media environment.

The alternative answer to the question is that knowledge about media choice will not converge. Following this line of reasoning, knowledge about media choice will continue to expand and diverge as media and uses evolve, as more contexts are explored, and as more primary goals are studied. Regardless of the answer to this question, this goal-based framework serves as a tool to identify where convergence is possible, and as an instrument to map the many variables, contexts, and goals as research evolves and develops.

\section{Conclusion}

Most of the CMC theories used in the IS and Communication literature today emerged in the late $1980 \mathrm{~s}$ and the 1990s, a period when digital technologies were only entering the mainstream. Digital media use was, for the majority of the population, new, and often experimental, infrequent, and deliberate. The media-choice theories that developed during that period reflect this newness, and some of the diversity found in this literature is the result of the ongoing changes in the media landscape that took place as theorizing and research continued. The theoretical expansion is evidence for productivity and pluralism, but also has some negative consequences, as both researchers and practitioners are often overwhelmed by the number and diversity of the theories. The goal-based framework developed here begins to address some of these consequences by demonstrating a common grounding of the highly divergent research on media choice. The framework is useful for promoting applied and theoretical academic research as well as for practitioners, and suggests new questions about the ultimate objective of media-choice research and theorizing.

\section{Acknowledgement}

We wish to thank Dr. Joe Walther for helpful discussions and for the idea to use goals as the basis of the proposed framework.

\section{References}

[1] Berger, C.R., "Communication: A Goal-directed, Planguided process", In D.R. Roskos-Ewoldsen and J.L. Monahan, eds., Communication and social cognition: Theories and methods. Lawrence Erlbaum Associates, 2007, 47-70.

[2] Carlson, J.R., and R.W. Zmud, "Channel Expansion Theory and the Experiential Nature of Media Richness Perceptions", The Academy of Management Journal 42(2), 1999, pp. 153-170.

[3] Carlson, P.J., and G.B. Davis, “An Investigation of Media Selection among Directors and Managers: From 'Self' to 'Other' Orientation”, MIS Quarterly 22(3), 1998, pp. 335362.

[4] Daft, R.L., and R.H. Lengel, "Information Richness - a New Approach to Managerial Behavior and Organization 
Design”, Research in Organizational Behavior 6, 1984, pp. 191-233.

[5] Daft, R.L., and R.H. Lengel, "Organizational Information Requirements, Media Richness and Structural Design”, Management Science 32(5), 1986, pp. 554-571.

[6] Daft, R.L., R.H. Lengel, and L.K. Trevino, "Message Equivocality, Media Selection, and Manager Performance: Implications for Information Systems", MIS quarterly 11(3), 1987, pp. 355-366.

[7] Dennis, A.R., R.M. Fuller, and J.S. Valacich, "Media, Tasks, and Communication Processes: A Theory of Media Synchronicity", MIS quarterly 32(3), 2008, pp. 575-600.

[8] Dillard, J.P., "Explicating the Goal Construct: Tools for Theorists", In J.O. Greene, ed., Message Production: Advances in Communication Theory. Routledge, New York, NY, 1997.

[9] Dillard, J.P., "The Goals-plans-action Model of Interpersonal Influence", In J.S. Seiter and R.H. Gass, eds., Perspectives on Persuasion, Social Influence, and Compliance Gaining. Pearson, Upper Saddle River, N.J.; Harlow, 2004, 185-206.

[10] Dillard, J.P., and D.H. Solomon, "Conceptualizing Context in Message-Production Research", Communication Theory 10(2), 2000, pp. 167-175.

[11] D'Urso, S.C., and S.A. Rains, "Examining the Scope of Channel Expansion: A Test of Channel Expansion Theory With New and Traditional Communication Media", Management Communication Quarterly 21(4), 2008, pp. 486-507.

[12] Fulk, J., J. Schmitz, and C.W. Steinfield, "A Social Influence Model of Technology Use.”, In J. Fulk and C.W. Steinfield, eds., Organizations and Communications Technology. Sage, Newbury Park, CA, 1990.

[13] Fulk, J., C.W. Steinfield, J. Schmitz, and J.G. Power, “A Social Information Processing Model of Media Use in Organizations", Communication Research 14(5), 1987, pp. 529.

[14] Greene, J.O., Message production: advances in communication theory, L. Erlbaum Associates, Mahwah, NJ, 1997.

[15] Hovorka, D., and K. Larsen, "Cfp: Knowing What We Know: Theory, Meta-Analysis and Review Minitrack", 2018. http://hicss.hawaii.edu/tracks-52/organizational-systems-andtechnology/\#knowing-what-we-know

[16] Jung, Y., and K. Lyytinen, "Towards an Ecological Account of Media Choice: A Case Study on Pluralistic
Reasoning While Choosing Email", Information Systems Journal 24(3), 2014, pp. 271-293.

[17] Katz, E., J.G. Blumler, and M. Gurevitch, "Uses and Gratifications Research", The Public Opinion Quarterly 37(4), 1973, pp. 509-523.

[18] Keck, K.L., and J.A. Samp, “The Dynamic Nature of Goals and Message Production as Revealed in a Sequential Analysis of Conflict Interactions", Human Communication Research 33(1), 2007, pp. 27-47.

[19] Kock, N., "Media Naturalness Theory: Human Evolution and Behaviour Towards Electronic Communication Technologies", In S. Roberts Craig, ed., Applied Evolutionary Psychology. Oxford University Press, USA, 2012, 381-398.

[20] Korzenny, F., "A Theory of Electronic Propinquity Mediated Communication in Organizations", Communication Research 5(1), 1978, pp. 3-24.

[21] LaRose, R., "The Problem of Media Habits", Communication Theory 20(2), 2010, pp. 194-222.

[22] Leonardi, P.M., "Crossing the Implementation Line: The Mutual Constitution of Technology and Organizing Across Development and Use Activities", Communication Theory 19(3), 2009, pp. 278-310.

[23] Marschak, J., "Economics of Inquiring, Communicating, Deciding", The American Economic Review 58(2), 1968, pp. $1-18$.

[24] Palomares, N.A., "The Goal Construct in Interpersonal Communication", In C.R. Berger, ed., Handbook of communication science. De Gruyter Mouton, Berlin, 2014, 77-100.

[25] Pervin, L.A., Goal concepts in personality and social psychology, Psychology Press, New York, NY, USA, 2015.

[26] Riemer, K., and S. Filius, "Contextualising Media Choice Using Genre Analysis", Business \& Information Systems Engineering 1(2), 2009, pp. 164-176.

[27] Riley, M.W., and J.W. Riley, "A Sociological Approach to Communications Research", Public Opinion Quarterly 15(3), 1951, pp. 445-460.

[28] Roberts, H.V., "The measurement of advertising results", The Journal of Business of the University of Chicago 20(3), 1947, pp. 131-145.

[29] Scissors, L.E., A.J. Gill, K. Geraghty, and D. Gergle, "In CMC We Trust: The Role of Similarity", CHI 2009, (2009). 
[30] Shachaf, P., and N. Hara, "Behavioral Complexity Theory of Media Selection: A Proposed Theory for Global Virtual Teams", Journal of Information Science 33(1), 2007, pp. 63-75.

[31] Shannon, C.E., and W. Weaver, The Mathematical Theory of Communication, The University of Illinois Press, Urbana, 1975.

[32] Sitkin, S.B., K.M. Sutcliffe, and J.R. Barrios-Choplin, "A Dual-Capacity Model of Communication Media Choice in Organizations", Human Communication Research 18(4), 1992, pp. 563-598.

[33] Stephens, K.K., "The Successive Use of Information and Communication Technologies at Work", Communication Theory 17(4), 2007, pp. 486-507.

[34] Stephens, K.K., D.M. Mandhana, J.J. Kim, X. Li, E.M. Glowacki, and I. Cruz, "Reconceptualizing Communication Overload and Building a Theoretical Foundation", Communication Theory 27(3), 2017, pp. 269-289.

[35] Stephens, K.K., and A.S. Sætre, "Media choice and ICT use", In L.D. Browning, A.S. Sætre, K.K. Stephens and J. Sørnes, eds., Information and communication technology in action: Linking theory and narratives of practice. 2004, $27-$ 35 .

[36] Stephens, K.K., J.O. Sørnes, R.E. Rice, L.D. Browning, and A.S. Sætre, "Discrete, Sequential, and Follow-Up Use of Information and Communication Technology by Experienced ICT Users", Management Communication Quarterly 22(2), 2008, pp. 197-231.

[37] Straub, D., and E. Karahanna, "Knowledge Worker Communications and Recipient Availability: Toward a Task Closure Explanation of Media Choice", Organization Science 9(2), 1998, pp. 160-175.

[38] Treem, J.W., and P.M. Leonardi, "Social Media Use in Organizations: Exploring the Affordances of Visibility, Editability, Persistence, and Association", Annals of the International Communication Association 36(1), 2013, pp. 143-189.

[39] Trevino, L.K., J. Webster, and E.W. Stein, "Making Connections: Complementary Influences on Communication
Media Choices, Attitudes, and Use", Organization Science 11(2), 2000, pp. 163-182.

[40] Vance Wilson, E., and Y. Lu, "Communication Goals and Online Persuasion: An Empirical Examination", Computers in Human Behavior 24(6), 2008, pp. 2554-2577.

[41] Venkatesh, Morris, Davis, and Davis, "User Acceptance of Information Technology: Toward a Unified View", MIS Quarterly 27(3), 2003, pp. 425.

[42] Walther, J.B., "Interpersonal Effects in ComputerMediated Interaction - a Relational Perspective", Communication Research 19(1), 1992, pp. 52-90.

[43] Walther, J.B., "Theories of Computer-Mediated Communication and Interpersonal Relations", In M.L. Knapp and J.A. Daly, eds., The SAGE Handbook of Interpersonal Communication. Sage, Thousand Oaks, CA, 2011, 443-479.

[44] Walther, J.B., "Social Information Processing Theory (CMC)", In The International Encyclopedia of Interpersonal Communication. John Wiley \& Sons, Inc., 2015.

[45] Walther, J.B., and N.N. Bazarova, "Validation and Application of Electronic Propinquity Theory to ComputerMediated Communication in Groups", Communication Research 35(5), 2008, pp. 622-645.

[46] Walther, J.B., and M.R. Parks, "Cues filtered out, cues filtered in", In M. Knapp and J. Daly, eds., Handbook of interpersonal communication. Sage, Thousand Oaks, CA, 2002, 529-563.

[47] Walther, J.B., B. Van Der Heide, L.M. Hamel, and H.C. Shulman, "Self-Generated Versus Other-Generated Statements and Impressions in Computer-Mediated Communication a Test of Warranting Theory Using Facebook", Communication research 36(2), 2009, pp. 229253.

[48] Webster, J., and L.K. Trevino, "Rational and Social Theories as Complementary Explanations of Communication Media Choices: Two Policy-Capturing Studies", Academy of Management Journal 38(6), 1995, pp. 1544-1572. 